\title{
Colchicine suppression of corneal healing after strabismus surgery
}

\author{
BEN ZION BIEDNER, LEONARD ROTHKOFF, LUDWIG FRIEDMAN, AND \\ CECELIA GELTMAN \\ From the Department of Ophthalmology, Soroka Medical Center, and Faculty of Health Sciences, \\ Ben Gurion University of the Negev, Beersheva, Israel
}

SUMMARY Two patients who had previously undergone uneventful operations for strabismus showed healing of corneal dellen and erosion after withdrawal of colchicine therapy. It is suggested that the exhibition of colchicine therapy for familial Mediterranean fever in these two cases was responsible for initial persistence of these two postoperative complications.

The occurrence of corneal dellen is not uncommon after rectus muscle surgery, especially with the limbal approach (Tessler and Urist, 1975). The condition is usually transient and leaves no permanent effect. We recently operated on 2 children with strabismus who were receiving prophylactic colchicine therapy for familial Mediterranean fever. They both developed persistent marginal corneal lesions which healed only with cessation of colchicine.

\section{Case reports}

\section{CASE 1}

In January 1974 a 6-year-old boy underwent bilateral medial rectus recession and bilateral inferior oblique recession for a $\mathrm{V}$ esotropia using a limbal approach. The postoperative course was uneventful. Owing to recurrent attacks of familial Mediterranean fever a prophylactic course of colchicine therapy, 3 tablets daily, was begun in August 1975. Because of residual esotropia a bilateral lateral rectus resection using a limbal approach was performed in February 1976. On the first postoperative day shallow saucer-like excavations were seen at the temporal margins of both corneas. Bilaterally the lesions formed an ellipse, $2 \mathrm{~mm} \times 1.5 \mathrm{~mm}$, parallel to the limbus, with their borders clearly defined. The wall on the corneal side was steep, while the limbal wall was sloping. The bases of the lesions appeared hazy and dry with definite fluorescein staining. A diagnosis of postoperative dellen was made.

Patching and chloramphenicol ointment were tried for 2 days without effect. The boy continued as an outpatient, receiving only chloramphenicol ointment twice daily. At 4 weeks after operation no change was seen in the dellen. Treatment was

Address for reprints: Dr B. Biedner, Department of Ophthalmology, Soroka Medical Center, Beersheva, Israel changed to chloramphenicol drops 3 times daily. At 8 weeks after operation the dellen still persisted and were approximately one-third the stromal thickness in depth (Fig. 1). Because of our suspicion that colchicine might be implicated in the delayed healing treatment with this drug was stopped. Within 3 days the dellen had completely healed except for a slight subepithelial haze, which has persisted until the present.

C.ASE 2

A 13-year-old boy had previously undergone a bilateral medial rectus recession in December 1967, using a limbal approach. The postoperative course was uneventful. In October 1975 treatment had been started with colchicine, 3 tablets daily, for

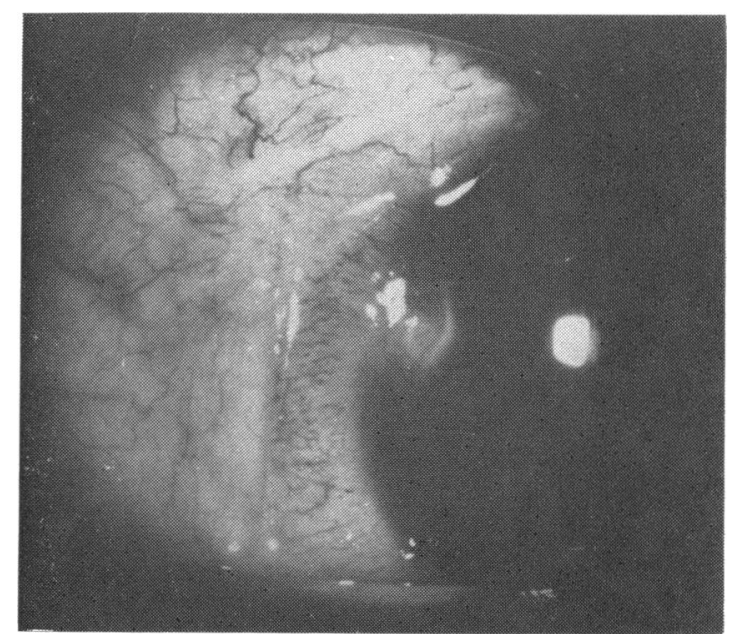

Fig. 1. Appearance of right cornea of case l eight weeks after surgery showing the hazy appearance of the persistent dellen 
prevention of attacks of familial Mediterranean fever. In December 1976 a bilateral lateral rectus resection was performed using a limbal approach for residual esotropia. On the first postoperative day a corneal erosion extending from 9 o'clock to 12 o'clock with fluorescein staining was present on the right cornea. The eye was treated with chloramphenicol ointment and patching, with no decrease in size or staining up to 3 weeks after operation. In consultation with the patient's paediatrician it was decided to discontinue the colchicine therapy. Healing of the corneal erosion was complete in 2 days without any residual defect.

\section{Discussion}

Colchicine is a well-documented antimitotic agent, arresting cell division in the metaphase. Cells with the highest rate of division are the most affected (Woodbury and Fingl, 1975). The effect of systemic colchicine in arresting corneal epithelium replication has previously been shown and has been used experimentally in studying corneal epithelial mitosis (Buschke et al., 1943).

Familial Mediterranean fever is a disease of unknown origin characterised by recurrent attacks of fever and polyserositis (Wolff, 1974). Prophylactic treatment with colchicine has been shown to decrease the incidence of these attacks (Goldstein and Schwabe, 1974). Its specific mode of action in this regard is still unknown, though its interference with microtubule assembly, phagocytosis, and degranulation may be involved (Dinarello et al., 1974). Some of the serious side effects noted have been azoospermia (Merlin, 1972), diarrhoea (Shanbrom and Rapoport, 1958), bone-marrow depression (Wallace, 1961), and chromosomal abnormalities (Ferreira and Buonicenti, 1968) apparently due to its effect on mitosis.

The limbal approach to muscle surgery has enjoyed popularity because of the reduction in scar tissue and easier access to the muscles (Von Noorden, 1968). However, swelling of limbal tissues may contribute to corneal dellen and other peripheral corneal inflammations (Aronson et al., 1970). These are usually transient, but permanent scarring and vascularisation have been reported (Baum et al., 1968). Dellen are particularly common after rectus muscle resections because of increased swelling of limbus tissues (Tessler and Urist, 1975).

In the 2 cases presented both had undergone uneventful extraocular muscle surgery before the institution of therapy with colchicine. Only after secondary surgery while receiving colchicine did peripheral dellen and a corneal erosion develop resistant to standard treatment. On cessation of colchicine healing was rapid. Since in dellen and erosions the integrity of the corneal epithelium and its ability to replicate are essential to the healing process (Duke-Elder and Leigh, 1965; Hogan and Zimmerman, 1962), it is not surprising that a potent antimitotic agent such as colchicine will delay repair.

It would seem prudent for patients undergoing operations for strabismus who are simultaneously receiving prophylactic colchicine therapy for familial Mediterranean fever either to abandon the limbal approach to the muscles or to discontinue the colchicine prior to surgery in consultation with the paediatrician or internist.

We wish to thank Mr David Grynspan for the photography, and Mrs Bilha Savell for technical assistance.

\section{References}

Aronson, S. B., Elliott, J. H., Moore, T. E., Jr, and O'Day, D. M. (1970). Pathogenic approach to therapy of peripheral corneal inflammatory disease. American Journal of Ophthalmology, 70, 65-90.

Baum, J. L., Mishima, S., and Boruchoff, S. A. (1968). On the nature of dellen. Archives of Ophthalmology, 79, 657662.

Buschke, W., Friedenwald, J. S., Fleischmann, W. (1943). Studies on the mitotic activity of the corneal epithelium. Johns Hopkins Hospital, Bulletin, 73, 143-168.

Dinarello, C. A., Wolff, S. M., Goldfinger, S. E., Dale, D. C., Alling, D. W. (1974). Colchicine therapy for familial Mediterranean fever. New England Journal of Medicine, 291, 934-937.

Duke-Elder, S., and Leigh, A. G. (1965). System of Ophthalmology, Vol. 8, part 2, pp. 867-868. Henry Kimpton: London.

Ferreira, N. R., and Buoniconti, A. (1968). Trisomy after colchicine therapy. Lancet, $2,1304$.

Goldstein, R. C., and Schwabe, A. D. (1974). Prophylactic colchicine therapy in familial Mediterranean fever. Annals of Internal Medicine, 81, 791-794.

Hogan, M. J., and Zimmerman, L. E. (1962). Ophthalmic Pathology, 2nd edn., p. 311. Saunders: Philadelphia.

Merlin, H. E. (1972). Azoospermia caused by colchicine: a case report. Fertility and Sterility, 23, 180-181.

Shanbrom, E., and Rapoport, L. (1958). Gastrointestinal complications of colchicine therapy in gout. Annals of Internal Medicine, 48, 655-660.

Tessler, H. H., and Urist, M. J. (1975). Corneal dellen in the limbal approach to rectus muscle surgery. British Journal of Ophthalmology, 59, 377-379.

Von Noorden, G. K. (1968). The limbal approach to surgery of the rectus muscles. Archives of Ophthalmology, 80, 94-97.

Wallace, S. L. (1961). Colchicine. American Journal of Medicine, 30, 439-448.

Wolff, S. M. (1974). Familial Mediterranean Fever (familial paroxysmal polyserositis). In Harrison's Principals of Internal Medicine, 7th edn., pp. 1068-1071. McGraw-Hill Book Co: New York.

Woodbury, D. M., and Fingl, E. (1975). Drugs employed in the therapy of gout. In The Pharmacological Basis of Therapeutics, 5th edn., pp. 350-352. Edited by L. S. Goodman and A. Gilman. Macmillan Publishing: New York. 